\title{
Conhecimento dos adolescentes sobre contraceptivos de uma escola pública no interior de Pernambuco
}

\author{
Adolescents knowledge about contraceptives in a public school in the interior of Pernambuco \\ Conocimientos de adolescentes sobre anticonceptivos en una escuela pública del interior de
}

Pernambuco

Recebido: 24/09/2021 | Revisado: 04/09/2021 | Aceito: 05/10/2021 | Publicado: 09/10/2021

\author{
Jéssica Maria Fragoso Cavalcante \\ ORCID: https://orcid.org/0000-0001-7822-5996 \\ Faculdade de Integração do Sertão, Brasil \\ E-mail: jessifragosoca@hotmail.com \\ José Luis Guilherme Fragoso Cavalcante \\ ORCID: https://orcid.org/0000-0002-1071-3632 \\ Faculdade de Integração do Sertão, Brasil \\ E-mail: luisguilherme_cavalcante@hotmail.com \\ Jullyana Maria Martins da Silva Soares \\ ORCID: https://orcid.org/0000-0001-5387-1844 \\ Faculdade de Integração do Sertão, Brasil \\ E-mail: jullyanamartins04@hotmail.com \\ Carlos Alberto Fragoso de Souza \\ ORCID: https://orcid.org/0000-0002-8388-4897 \\ Universidade Federal do Agreste de Pernambuco, Brasil \\ E-mail: carlos_fragoso1@hotmail.com \\ Erika Valente de Medeiros \\ ORCID: https://orcid.org/0000-0001-5543-9414 \\ Universidade Federal do Agreste de Pernambuco, Brasil \\ E-mail: erika.valente@ufape.edu.br \\ João Paulo da Silva Teixeira \\ ORCID: https://orcid.org/0000-0002-1282-9903 \\ Faculdade de Integração do Sertão, Brasil \\ E-mail: joaopaulo77teixeira@gmail.com \\ Gabriela Cavalcante da Silva \\ ORCID: https://orcid.org/0000-0003-3390-6645 \\ Faculdade de Integração do Sertão, Brasil \\ E-mail: gcavalcante1988@gmail.com
}

\begin{abstract}
Resumo
Métodos contraceptivos ou anticoncepcionais são todos os recursos utilizados por homens e mulheres para evitar gravidez e doenças sexualmente transmissíveis. Um dos principais métodos contraceptivos de difusão mundial são os contraceptivos hormonais orais, conhecidos como pílulas anticoncepcionais, estes têm sido amplamente utilizados pela população feminina. Além da ação contraceptiva, essa classe de medicamentos apresenta outras finalidades terapêuticas como, tratamento de cistos ovarianos, tratamento do câncer ovariano e endometrial, entre outros. Com base nisso pretendeu-se então conhecer o perfil de comportamento dos adolescentes acerca do tema e avaliar o nível de conhecimento sobre métodos contraceptivos. Como instrumento de pesquisa foi aplicado um questionário no formato eletrônico abordando questões acerca dos métodos contraceptivos, com alunos do ensino médio de rede pública com idade entre 14 e 19 anos. Os resultados do trabalho contaram com a participação de 127 estudantes, dentro desse grupo $96(75,6 \%)$ pessoas eram do sexo feminino e 31(24,4\%) do sexo masculino, ainda foi evidenciado que $55(43,3 \%)$ dos adolescentes não tem orientação no ambiente familiar sobre educação e orientação sexual, além de que $48(37,8 \%)$ dos entrevistados afirmam não conhecer os riscos das possíveis interações medicamentosas, enquanto $46(36,2 \%)$ acreditam que não possam desencadear doenças. Conclui-se que os adolescentes possuem algumas informações sobre os métodos contraceptivos, porém, superficiais, ficando explícito a necessidade de aprimorar à divulgação de conhecimento a respeito da saúde sexual.
\end{abstract}

Palavras-chave: Saúde pública; Anticoncepcionais; Educação em saúde; Adolescência; Contraceptivos.

\begin{abstract}
Contraceptive methods or contraceptives are all resources used by men and women to prevent pregnancy and sexually transmitted diseases. One of the main contraceptive methods of worldwide diffusion are the oral hormonal contraceptives, known as birth control pills, these have been widely used by the female population. In addition to the
\end{abstract}


contraceptive action, this class of drugs has other therapeutic purposes such as treatment of ovarian cysts, treatment of ovarian and endometrial cancer, among others. Based on this, it was intended then to know the behavior profile of adolescents on the subject and assess the level of knowledge about contraceptive methods. As a research instrument, an electronic questionnaire was applied, addressing issues about contraceptive methods, with public high school students aged between 14 and 19 years. The results of the work had the participation of 127 students, within this group 96 (75.6\%) people were female and 31 (24.4\%) were male, it was also evidenced that $55(43.3 \%)$ of the adolescents do not have guidance in the family environment about education and sexual orientation, and 48 (37.8\%) of the interviewees claim not to know the risks of possible drug interactions, while $46(36.2 \%)$ believe that they cannot trigger diseases. It is concluded that teenagers have some information about contraceptive methods, however, it is superficial, making clear the need to improve the dissemination of knowledge about sexual health.

Keywords: Public health; Contraceptives; Health education; Adolescence; Contraceptives.

\section{Resumen}

Los métodos anticonceptivos o anticonceptios son todos los recursos utilizados por hombres y mujeres para prevenir el embarazo y las enfermedades de transmisión sexual. Uno de los principales métodos anticonceptivos de difusión mundial son los anticonceptivos hormonales orales, conocidos como píldoras anticonceptivas, estos han sido ampliamente utilizados por la población femenina. Además de la acción anticonceptiva, esta clase de fármacos tiene otras finalidades terapéuticas como el tratamiento de quistes ováricos, tratamiento de cáncer de ovario y endometrio, entre otros. En base a esto, se pretendió luego conocer el perfil de comportamiento de los adolescentes sobre el tema y evaluar el nivel de conocimiento sobre los métodos anticonceptivos. Como instrumento de investigación, se aplicó un cuestionario electrónico, abordando temas sobre métodos anticonceptivos, con estudiantes de secundaria pública de entre 14 y 19 años. Los resultados del trabajo contaron con la participación de 127 estudiantes, dentro de este grupo $96(75,6 \%)$ personas eran mujeres y $31(24,4 \%)$ hombres, también se evidenció que $55(43,3 \%)$ de los adolescentes no cuentan con orientación en el entorno familiar sobre educación y orientación sexual, y 48 (37,8\%) de los entrevistados afirman no conocer los riesgos de posibles interacciones medicamentosas, mientras que $46(36,2 \%)$ creen que no pueden desencadenar enfermedades. Se concluye que los adolescentes cuentan con alguna información sobre los métodos anticonceptivos, sin embargo, es superficial, dejando clara la necesidad de mejorar la difusión del conocimiento sobre salud sexual.

Palabras clave: Salud pública; Anticonceptivos; Educación para la salud; Adolescencia; Anticonceptivos.

\section{Introdução}

A adolescência constitui uma fase de desenvolvimento caracterizada por profundas transformações a nível físico, psicológico, afetivo, social e familiar, é um período de transição entre a infância e a idade adulta. A progressiva maturação fisiológica é normalmente acompanhada pela súbita descoberta de novas relações e experiências, de ordem afetiva e sexual, muitas vezes geradoras de intensos conflitos, assumindo um aspecto importante, pois tais mudanças ocorrem de forma rápida, profunda e marcante, podendo interferir de forma positiva ou negativa para o resto da vida do indivíduo (Moura, 2015).

Segundo a Organização Mundial da Saúde (OMS), a adolescência é compreendida pela faixa etária de 10 a 19 anos (Trabbold et al., 2016). Período este marcado por grandes alterações biológicas, emocionais e sociais, por este motivo é uma fase de grande vulnerabilidade (Mesquita et al., 2017). Além disso, essa fase traz incertezas, questionamentos e desejos, levando-os ao início cada vez mais precoce das relações sexuais o que pode torná-los mais susceptíveis à uma gravidez indesejada e às infecções sexualmente transmissíveis (ISTs) (Gonçalves et al., 2016).

Os jovens comumente têm sua primeira relação sexual, muitas vezes de forma desprotegida, seja por desconhecimento dos métodos de prevenção ou mesmo por, embora tendo ciência de tais métodos, resolvem ainda assim assumir uma conduta de risco (Spindola et al., 2020). A taxa global de gravidez na adolescência é estimada em 46 nascimentos por 1.000 jovens, enquanto as taxas de gravidez na adolescência na América Latina e no Caribe constituem as segundas mais altas do mundo, estimadas em 66,5 nascimentos por 1.000 jovens de 15 a19 anos. No Brasil, embora seja observada uma queda de $33 \%$ na taxa de gravidez na adolescência no período de 2000 a 2016, ainda é observada uma frequência de 58,7/1.000, superior à taxa média das Américas, de 48,6/1000 (Paho \& Who, 2018).

A relevância do tema está explícito na elevada taxa de nascimentos entre adolescentes de 15 a 19 anos, aumentando os riscos à saúde de recém-nascidos e para as jovens mães (Vignoli, 2017; Who, 2018), situação que reforça 
tanto a importância de que, seja intensificado o diálogo acerca da sexualidade entre pais e filhos, como também que sejam promovidas ações educativas que visem desmitificar tabus sobre o assunto e orientem os adolescentes quanto aos riscos de uma relação sexual desprotegida e o planejamento familiar.

A não utilização dos contraceptivos orais e do preservativo possivelmente terão como consequências uma gestação não desejada, aumentando assim as chances de abortos inseguros e ISTs (Ferreira, 2019). Segundo Miranda et al. (2016), estudos demonstram, quanto ao comportamento sexual dos adolescentes, que a maioria, mesmo conhecendo os métodos contraceptivos, inicia a vida sexual sem proteção e, no seguimento da atividade sexual, quase $30 \%$ não se protegem, tanto na contracepção como contra as IST/AIDS.

A escolha do método contraceptivo é influenciada por vários fatores, incluindo o acesso a diferentes métodos, características pessoais do adolescente, do seu parceiro, entre outros. O conhecimento sobre vários métodos contraceptivos e o desejo de experimentá-los para uma maior adaptação são essenciais para o sucesso da contracepção (Apter, 2018). Diante disso, pretendeu-se então conhecer o perfil de comportamento dos adolescentes acerca do tema e avaliar o nível de conhecimento sobre métodos contraceptivos.

\section{Metodologia}

Trata-se de um levantamento de campo no Município Tabira - PE localizado na mesorregião do sertão, a 413,5 km de distância da capital, Recife, na escola EREM (Escola de Referência em Ensino Médio) Professora Carlota Breckenfeld. A referida instituição de ensino conta com 310 alunos do ensino médio, desses, 127 alunos responderam ao questionário correspondendo a $92,028 \%$ do $\mathrm{N}$ amostral, considerando parâmetros de erro de 5\%, nível de confiança de $95 \%$. Além do cálculo amostral, foram utilizados como parâmetro de critério de inclusão e exclusão a idade, inferior a 14 anos de idade não poderiam participar da pesquisa e nem maior dos 19.

O questionário semiestruturado foi baseado utilizando temas de artigos da Biblioteca virtual em saúde do ministério da saúde (BVSMS) os quais tratavam das principais debilidades de conhecimento acerca dos contraceptivos. Elegendo-se assim dezenove questões objetivas, confeccionadas em formulário eletrônico (Google Forms).

A primeira seção deste constava do Termo de Consentimento Livre e Esclarecido (TCLE) e do Termo de Assentimento livre e esclarecido (TALE) estes foram assinados pelos responsáveis de forma online e pelos alunos respectivamente. O link do questionário foi enviado a diretora da escola e repassado para os estudantes, via aplicativo de mensagens, e ficou aberto no período de janeiro a fevereiro de 2021. O início da coleta de dados se deu após aprovação pelo Comitê de Ética em Pesquisa da Faculdade de Integração do Sertão - FIS, número do CAAE: 03124918.9.0000.8267 e número do parecer: 3.624.235.

Os dados obtidos na pesquisa foram interpretados e processados na forma de gráficos utilizando o software GraphPad Prism versão 8.0.

\section{Resultados e Discussão}

A população foi composta por 127 estudantes, distribuídos nas seguintes faixa-etárias: $125(98,4 \%)$ tinham entre 15 a 20 anos; 2 (1,6\%) tinham 14. Dentro desse grupo 96 (75,6\%) pessoas eram do gênero feminino e 31 (24,4\%) do gênero masculino (Figura 1). 
Figura 1 - Distribuição dos participantes da pesquisa segundo gênero.

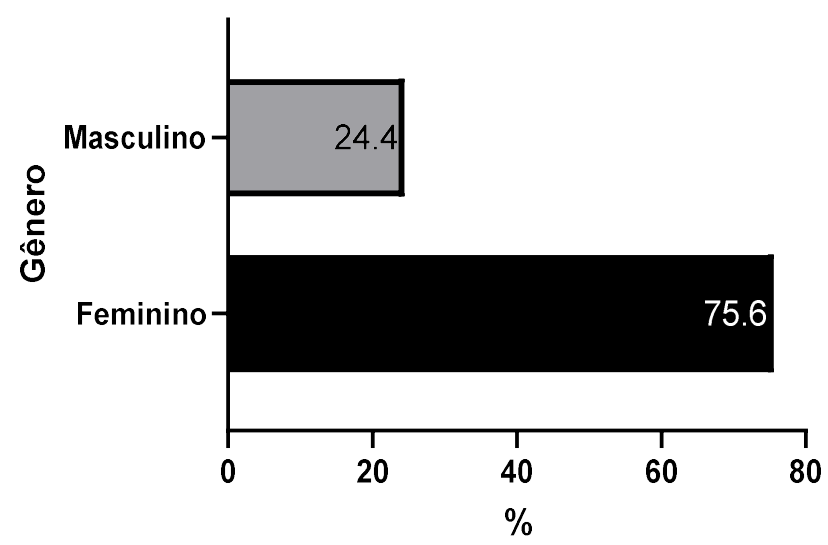

Fonte: Autores (2021).

Conforme ilustra a Figura 2, $110(86,6 \%)$ dos participantes do estudo disseram já ter vivenciado alguma ação educativa com o tema voltado para o planejamento familiar (uso de métodos contraceptivos), enquanto 17 (13,4\%) alegaram que não tiveram acesso a esse tipo de educação.

Figura 2 - Presença dos participantes em ações educativas de planejamento familiar.

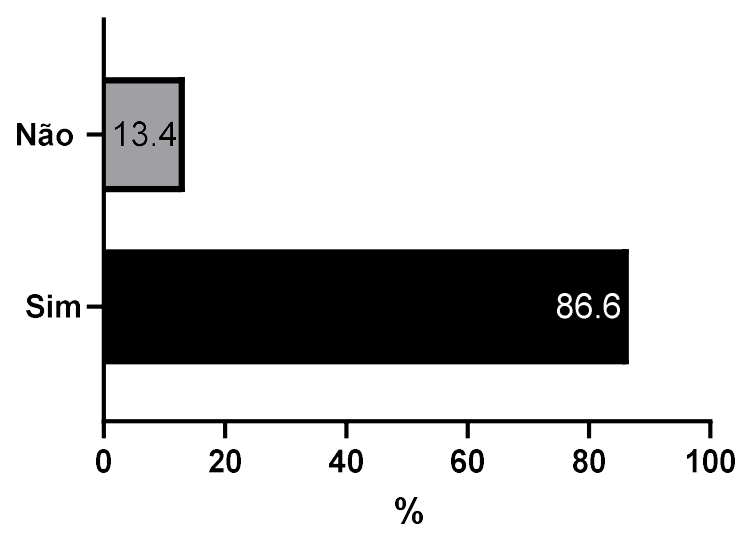

Fonte: Autores (2021).

Os métodos contraceptivos mais conhecidos pelos adolescentes deste estudo foram o preservativo masculino, 124 (97,6\%), e a pílula anticoncepcional, 121 (95,3\%) (Figura 3), corroborando com estudos realizados por Ramos et al. (2018) onde verificou-se que dentre adolescentes de escola pública do Maranhão $184(92,5 \%)$ referiram ter informações acerca e conhecer a camisinha masculina. Observou-se diferença quanto ao conhecimento dos métodos contraceptivos em relação ao sexo. É sabido que o conhecimento acerca dos diferentes métodos contraceptivos pelos adolescentes vem aumentando, assim como é consenso que os métodos mais conhecidos pelos jovens brasileiros são, fundamentalmente, a camisinha masculina e a pílula contraceptiva (Borges et al., 2016) 
Figura 3 - Conhecimento dos participantes da pesquisa sobre métodos contraceptivos.

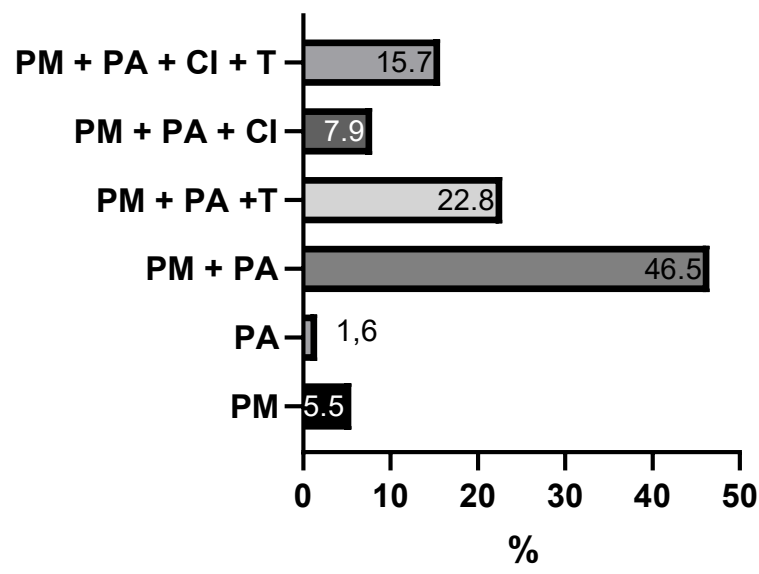

PM - Preservativo masculino; PA - Pílula anticoncepcional; CI - Coito interrompido; T - Tabelinha. Fonte: Autores (2021).

Os ACOs (anticoncepcionais orais) inibem a ovulação através de hormônios sintéticos de estrógeno e progesterona. Essa inibição ocorre através da supressão de fatores relacionados à adeno hipófise na liberação do FSH (hormônio folículo estimulante) e do LH (hormônio luteinizante). O progestógeno é o que diferencia cada fórmula, e age no espessamento do muco cervical, dificultando assim a passagem dos espermatozoides (Borges et al., 2015).

As pílulas de primeira geração, ou seja, as mais antigas eram compostas de estrogênio e progestógeno, essas pílulas apresentavam muitos efeitos adversos como cefaleia intensa e náuseas, e por isso não são mais utilizadas. As de segunda geração apresentam o etinistradiol em doses de 30 a $50 \mu \mathrm{g}$ além do levonorgestrel, e ainda são utilizadas por algumas mulheres, onde são distribuídas no Sistema Único de Saúde (SUS). As de terceira geração apresentam o etinistradiol em doses de $30 \mu \mathrm{g}$ ou menos e progestógenos que são as mais utilizadas (Dombrowski et al., 2013).

Os métodos de barreira e os hormonais também foram os mais citados por adolescentes de ambos os sexos de escola pública em Minas Gerais, com frequência de 94,4\% para o preservativo masculino e 83,1\% para os contraceptivos hormonais (Vieira et al., 2021). Corroborando também com esses achados, Kara et al. (2019), no seu estudo com 374 universitárias da St John's University, Dodoma - Tanzânia, citaram principalmente os preservativos (88,0\%) e os anticoncepcionais orais (83,5\%) como os métodos mais conhecidos.

Chama a atenção o fato de os adolescentes não terem citado métodos contraceptivos reversíveis de longa duração, como os implantes e o dispositivo uterino com hormônio. Este resultado também instiga a propor-se outros estudos considerando não apenas o conhecimento dos adolescentes, mas as expectativas e possibilidades de maior e mais fácil acesso aos métodos contraceptivos considerados seguros e adequados para o adolescente (Vieira et al., 2021).

Dentre os participantes do presente estudo 108 (85\%) disseram acreditar que o uso de medicamentos pode causar alterações no efeito do anticoncepcional, enquanto 19 (15\%) acreditam que não ocorre interferência quando é feito o consumo de vários tipos de medicações simultaneamente. Além de apresentar efeitos colaterais relacionados apenas ao uso da pílula contraceptiva, está pode apresentar outros efeitos advindos do uso de outros medicamentos, caracterizado como uma interação medicamentosa, acarretando, por vezes, na ineficácia do método (Braga, 2016; Acácio, 2018).

As interações medicamentosas de um modo geral e com a contracepção de emergência e hormonal agem por dois meios: sinergismo, ocorre com a potencialização do efeito da contracepção de emergência devido ao uso de outro medicamento que apresenta mecanismo de ação distinto, porém, com o mesmo efeito. A outra forma é denominada de antagonismo, que se caracteriza pela inibição do efeito, por competição ao alvo quimioterápico, e na ineficácia do método (Souza, 2015). Também se observa nas interações medicamentosas o aditivismo, que consiste apenas na soma dos efeitos das 
drogas individuais, que são recomendadas também em uso comercial de coquetéis de fármacos pela OMS (Foucquier \& Guedj, 2015).

Como mostra a Figura 4, 79 (62,2\%) dos entrevistados afirmam conhecer os riscos das possíveis interações medicamentosas e 48 (37,8\%) afirmam não conhecer os riscos, o que evidencia a necessidade de mais aprendizado na área.

Figura 4 - Ciência dos participantes da pesquisa sobre interações medicamentosas com anticoncepcionais.

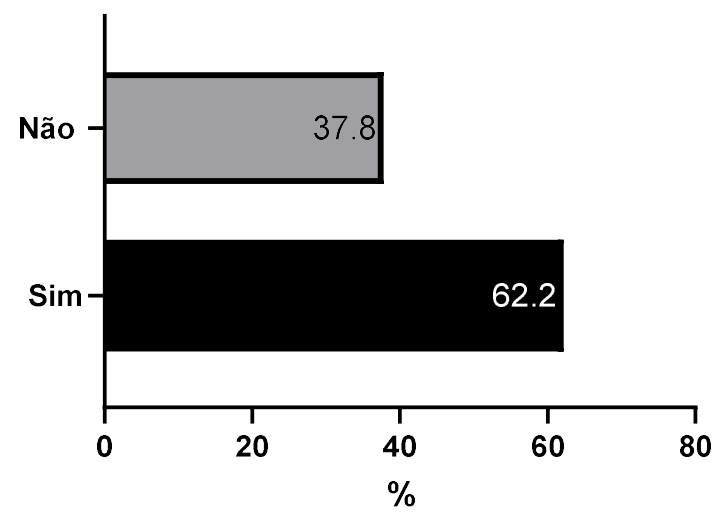

Fonte: Autores (2021).

Fármacos como os barbitúricos, fenitoína, rifampicinas, antirretrovirais, isotretinoína, oxcarbazepina, carbamazepina, primidona, clobazam e antibióticos das classes das penicilinas, tetraciclinas, e cefalosporinas quando administrados juntamente com a contracepção de emergência, aceleraram o metabolismo da mesma, reduzindo sua eficácia. A amiodarona e a teriflunomida aumentam a concentração do CE (contracepção de emergência) no organismo, podendo aumentar a probabilidade de desenvolvimento dos efeitos colaterais (Melcon, 2014).

Diante dos efeitos colaterais, consequências sociais, interações medicamentosas, torna-se fundamental a educação por parte dos pais, da escola e da atenção profissionais da saúde, como os farmacêuticos, que estão ligados diretamente a este contexto. Um ponto positivo neste contexto seria a educação e orientação sexual domiciliar, neste estudo, ainda foi evidenciado que $55(43,3 \%)$ dos adolescentes não tem essa orientação no ambiente familiar, enquanto 72 (56,7\%) disse que tem esse contato com familiares (Figura 5).

Figura 5 - Acesso à orientação sexual domiciliar pelos participantes.

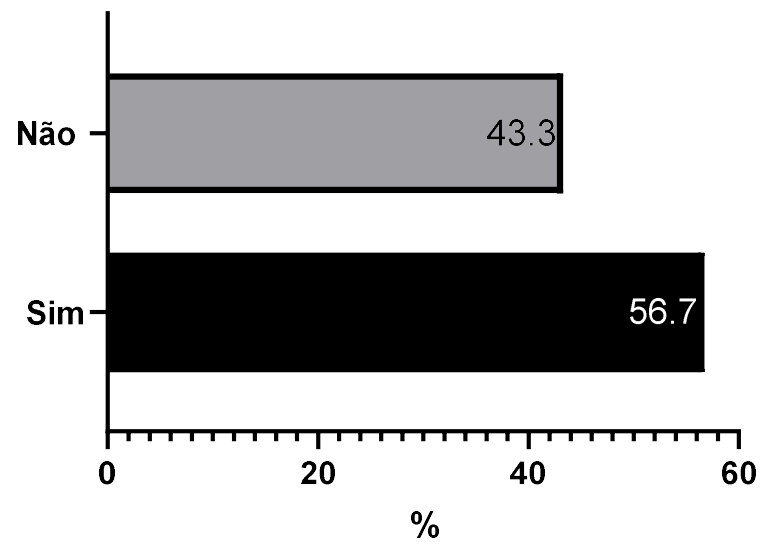

Fonte: Autores (2021). 
Dos participantes da pesquisa $96(75,6 \%)$ relataram não fazer uso de métodos contraceptivos, $15(11,8 \%)$ afirmaram utilizar do método anticoncepcional oral, $15(11,8 \%)$ relataram fazer uso do preservativo e $1(0,8 \%)$ disse utilizar como meio de contracepção a pílula do dia seguinte, Figura 6.

Figura 6 - Utilização dos Contraceptivos pelos participantes da pesquisa.

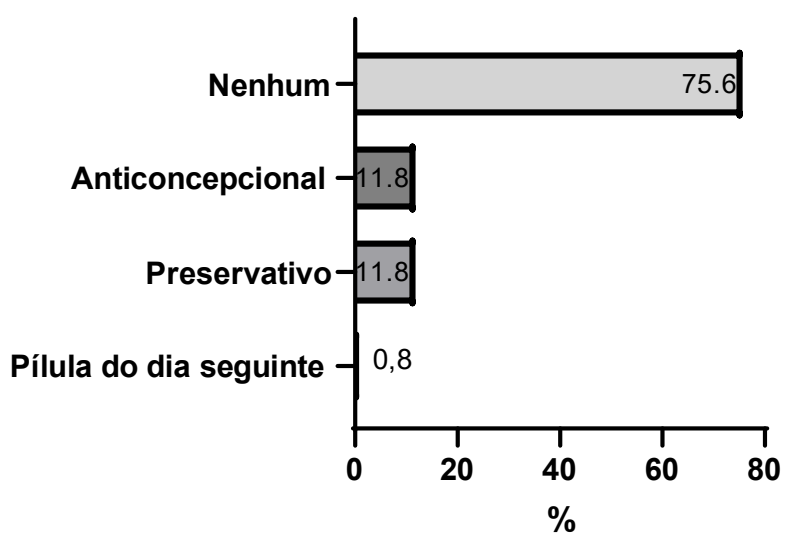

Fonte: Autores (2021).

Independentemente do método escolhido, os adolescentes devem ser aconselhados sobre a importância do uso de preservativos para reduzir o risco de aquisição de IST (Todd \& Black, 2020). Outro estudo mostra que o preservativo masculino é o método mais conhecido contra IST e gravidez entre os jovens, apesar do seu uso consistente não ser frequente, principalmente nas relações eventuais e não programadas (Ramos et al., 2018). A maioria dos participantes afirmaram conhecer a maneira correta de utilizar os métodos contraceptivos 89 (70,1\%). Existe uma margem significativa de dificuldade de distinguir se os anticoncepcionais podem desencadear algum tipo de doença entre os participantes da pesquisa, onde $81(63,8 \%)$ afirmaram que os anticoncepcionais podem desencadear alguma doença e 46 (36,2\%) acreditam que não possam desencadear doenças.

Thapa et al. (2018) evidenciaram como benefícios de utilização de contraceptivos por suas entrevistadas da cidade sub-metropolitana do Nepal, evitar gravidez indesejada (79,3\%), manter espaçamento entre nascimentos (71,5\%), limitar número de partos (68,9\%), melhorar a saúde da mãe e do filho $(59,6 \%)$ e diminuir o ônus econômico da família (46,6\%) foram identificados como os principais benefícios da contracepção. Isso pode resultar do fato de que mulheres instruídas têm mais probabilidade de estar a par dos anticoncepcionais disponíveis e são mais propensas a reconhecer os impactos positivos que os anticoncepcionais têm em suas vidas (Nyarko, 2015).

Os jovens não têm buscado os profissionais de saúde para receberem informações sobre métodos contraceptivos, o que constitui um ponto negativo para a saúde dos adolescentes, tendo em vista um estudo, realizado em Gana (Nyako, 2015). Destarte, considera-se que os profissionais de saúde possuem um papel fundamental nesse processo, e, por isso, devem estar promovendo constantemente ações de saúde que gerem troca de informações sobre saúde sexual e reprodutiva, a fim de empoderar os adolescentes quanto às boas práticas contraceptivas (Queiroz et al., 2017). Os adolescentes que iniciaram a vida sexual e procuraram algum tipo de acompanhamento médico (ginecologista/ urologista) foram 6 (4,7\%).

Segundo ilustra a Figura 7, a maioria dos participantes tem o conhecimento quanto ao local onde conseguir métodos contraceptivos pelo SUS de forma gratuita 80 (63\%). A Estratégia Saúde da Família (ESF) têm como responsabilidade a assistência à saúde do adolescente bem como o acesso aos métodos contraceptivos e ao planejamento familiar, contudo, a frequência desses adolescentes, nessas unidades, é pouco perceptível, o que é presente é a real necessidade 
de práticas voltada para esse público com o intuito de trazê-los as unidades de saúde para terem acesso a esses programas e uma qualidade de vida futura melhor (Rodrigues et al., 2017).

Figura 7 - Conhecimento sobre distribuição de contraceptivos pelo SUS.

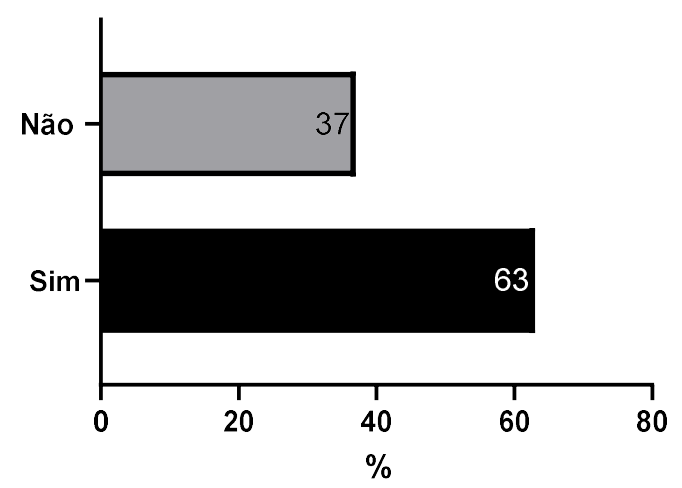

Fonte: Autores (2021).

O planejamento familiar é o direito que toda pessoa tem de optar por ter ou não ter filhos, por meio de uma assistência especializada e com informação. Estas são ofertadas por intermédio da atenção básica durante o programa de planejamento familiar. Englobar os adolescentes no planejamento familiar é uma tarefa de grande importância, uma vez que neste período estão despertando em si a sexualidade (Silva et al., 2016)

Dentre os participantes 79 (62,2\%) afirmaram que a escola oferece algum tipo de informação sobre sexualidade, o que evidencia a importância de uma maior abordagem sobre o tema nas escolas. É importante aproveitar oportunidades de palestras em escolas, para promover o conhecimento e encorajar a adoção de hábitos e práticas sexuais saudáveis, que assegurem riscos mínimos à saúde sexual e reprodutiva de adolescentes (Franco et al., 2020). Informação e comunicação devem ser duas aliadas na prevenção e na promoção da saúde, evitando a disseminação de informações equivocadas.

Salienta-se que tanto na escola, quanto na atenção básica, a partir de atividades de educação em saúde, a informação e a autonomia dos adolescentes sejam fortalecidas a fim de que suas escolhas por práticas sexuais seguras sejam feitas de maneira consciente (Ferreira et al, 2019). Neste estudo, as principais fontes de informação, sobre métodos contraceptivos, referidas pelos adolescentes são internet 59 (46,5\%), palestras $37(29,1 \%)$, amigos $6(4,7 \%)$, por profissionais de saúde 23 $(18,1)$, por parceiros (as) $2(1,6 \%)$ (Figura 8$)$. 
Figura 8 -Fonte de informações sobre contraceptivos.

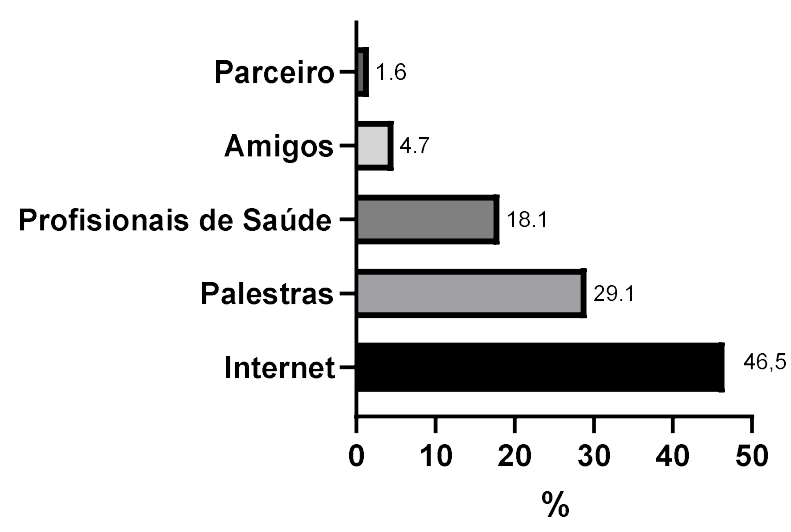

Fonte: Autores (2021).

Esse achado difere de trabalho estruturado no estado do Maranhão, realizado em escolas públicas e particulares, a maioria dos adolescentes relatou que a principal fonte de informação é a escola (71,0\%) e em segundo lugar, meios de comunicação em massa (46,8 \%) (Portela \& Albuquerque, 2014). Divergindo desses achados, Borges (2016) relatou em seu estudo com universitárias de medicina de uma faculdade de São José do Rio Preto no estado de São Paulo, que o conhecimento necessário sobre a sexualidade, chega ao aluno por meio de conversas informais com colegas e pela mídia, e pela via da educação sexual.

A frequência de adolescentes que já engravidou ou conhece alguém que engravidou na adolescência, como base nos adolescentes participantes da pesquisa desse trabalho evidenciaram que, $104(81,9 \%)$ disseram que conhecem alguém que engravidou na adolescência ou já engravidou e $23(18,1 \%)$ disseram não ter conhecimento sobre adolescentes que engravidaram. Existe um questionamento sobre relação entre os anticoncepcionais orais e a esterilidade da mulher e os estudantes entrevistados para esse trabalho mostraram seus pontos de vista a respeito do tema, 44 (34,6\%) disseram que acreditam que os anticoncepcionais possam causar a esterilidade da mulhere $83(65,4 \%)$ disseram que não.

A busca dos adolescentes por métodos contraceptivos, dentre eles o método de emergência, é frequente. Este método também conhecido como "pílula do dia seguinte" é um medicamento utilizado após a relação sexual afim de evitar gravidezes indesejadas. Seu uso expandiu-se consideravelmente nos últimos anos, por ser um medicamento de fácil acesso, e com dispensabilidade da receita médica, assim, culminando no uso irracional deste método entre adolescentes (Cavalcante, 2016). Já é sabido que a pílula do dia seguinte não tem a mesma segurança quando utilizado de maneira inadequada, de forma desordenada e mais de duas vezes ao ano; 49 (8,6\%) adolescentes afirmaram acreditar que a pílula é segura, enquanto 78 $(61,4 \%)$ acreditam que não, assim como está ilustrado na Figura 9. 
Figura 9 - Ciência sobre a segurança no uso de pílula do dia seguinte.

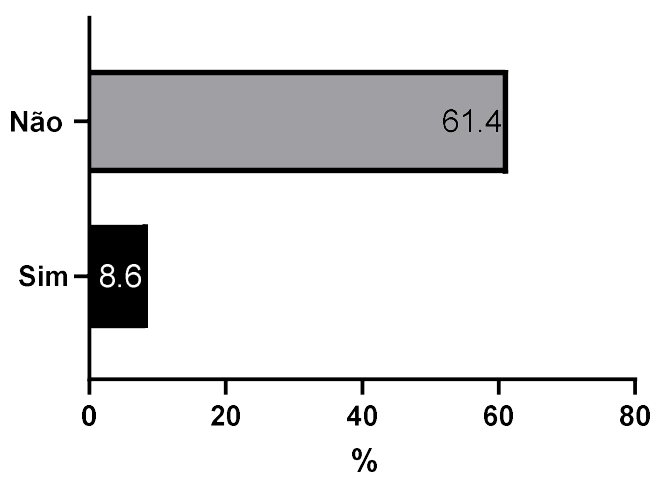

Fonte: Autores (2021).

A pílula anticonceptiva de emergência (AE), é composta por uma alta dosagem de hormônio (Levonorgestrel 1,5 mg, mais utilizada) considerada como uma "bomba hormonal", que age diretamente sob o mecanismo de secreção do hormônio folículo estimulante (FSH) e do hormônio luteinizante (LH), que impede a ovulação e consequentemente a fecundação (Braga et al., 2016). Diferentemente das pílulas anticoncepcionais convencionais, o contraceptivo de emergência não pode ser utilizado rotineiramente, como substituto de outro método anticoncepcional de rotina, pois, pode ter sua eficácia comprometida (SILVA et al., 2017). Por apresentar uma concentração de hormônios dez vezes mais alta que os métodos anticoncepcionais de rotina, o uso abusivo da "pílula do dia seguinte" pode conferir reações adversas, como o aumento de 2 a 6 vezes o risco de eventos tromboembolísticos e hemorragia vaginal (Acácio, 2018).

$\mathrm{O}$ conhecimento dos adolescentes sobre a $\mathrm{AE}$ normalmente se resume apenas a saber de sua existência e que isto não se traduz em saber onde, como e em quais situações utilizá-la, já que muitos dos respondentes utilizavam o método de forma incorreta. Aliado a isto, ainda se tem que, em geral, as principais fontes de informação sobre o método são os amigos, a escola e os meios de comunicação, sendo necessária uma melhor abordagem dos serviços de saúde nessa situação (Leite et al., 2020).

Fonteles et al. (2016) afirmam que grande parte das mulheres que fazem uso da contracepção de emergência pertence às classes média e baixa. Sendo assim, 77,8\% têm renda inferior a três salários mínimos, o que pode justificar certa dificuldade para aquisição do medicamento correto, bem como acompanhamento adequado do ginecologista. Estudos realizados nos estados de Pernambuco e São Paulo, com adolescentes com idades entre 14 e 19 anos de idade, mostrou uma taxa de utilização da anticoncepção de emergência de 27,8\% e 26,20\%, respectivamente (Gomes, 2016).

Na pesquisa de Zanini (2017), 45,3 \% das adolescentes entrevistadas, já haviam utilizado a contracepção de emergência. Isto causa bastante preocupação aos órgãos de saúde, pois, o uso desse medicamento tem sido feito de forma indiscriminada e errônea. $\mathrm{O}$ déficit de conhecimento dos adolescentes a respeito dos métodos contraceptivos, da prevenção de infecções sexualmente transmissíveis (IST) e das questões sobre a sexualidade persiste e representa um problema atual e pertinente (Almeida et al., 2017).

Assim, há a necessidade de ações voltadas para a saúde da mulher, em especial, dos adolescentes, no que diz respeito ao planejamento reprodutivo (Santos et al., 2018). Para tanto, faz-se indispensável o cuidado multiprofissional e interdisciplinar com o objetivo de romper paradigmas incorporados a valores socioculturais e atender às necessidades específicas deste grupo populacional, especialmente no que tange às questões relacionadas à sexualidade e à reprodução (Ferreira et al., 2019). 


\section{Conclusão}

Constatou-se que não houve nenhuma limitação quanto a abordagem do assunto, no entanto houve uma certa limitação na execução da pesquisa em vista que se tratou de questionário eletrônico e nem todos tinham acesso à internet nesse período de pandemia. Os adolescentes possuem informações sobre os métodos contraceptivos, principalmente, o preservativo masculino e a pílula anticoncepcional, porém por vezes e eventualmente superficiais a respeito de outros métodos contraceptivos, da prevenção de IST, da gravidez na adolescência e o uso adequando de anticoncepcionais convencionais e de emergência. Deve-se considerar a necessidade de aprimorar à divulgação de conhecimento aos adolescentes a respeito da saúde sexual, por ser um tema amplo e complexo, tais informações devem ser repassadas de forma contínua, em articulação com as escolas, as famílias e a comunidade, além de envolver todos os elementos que compõem a rede de apoio social aos adolescentes.

\section{Referências}

Acácio, A. J. M., Magalhães, E. P., \& Sampaio, T. L. (2018). Contraceptivos de emergência Avaliação do nível de informação de clientes de uma farmácia em Fortaleza: O nível de informação acerca de contraceptivos de emergência. Revista Diálogos Acadêmicos, 7(2).

Almeida, A. P. F., \& Assis, M. M. (2017). Efeitos colaterais e alterações fisiológicas relacionadas ao uso de contínuo de anticoncepcionais hormonais orais. Revista eletrônica Atualiza Saúde, 5 (5), 85-93.

Almeida, R. A. A. S., Corrêa R. G. C. F et al. (2017). Conhecimento de adolescentes relacionados às doenças sexualmente transmissíveis e gravidez. Revista Brasileira de Enfermagem, 70 (5), 1087-1094. https://doi.org/10.1590/0034-7167-2016-0531

Apter, D. (2018). Contraception options: aspects unique to adolescent and young adult. Best Practice \& Research Clinical Obstetrics \& Gynaecology, 48, 115-27. https://doi: 10.1016 / j.bpobgyn.2017.09.010.

Borges, A. L. V. et al. (2016). ERICA: início da vida sexual e contracepção em adolescentes brasileiros. Revista de Saúde Pública, 50, 1-11. https://doi:10.1590/S01518-8787.2016050006686

Borges, M. C. (2016). Knowledge on the effects of hormonal contraceptives by students of health area. Revista Baiana de Enfermagem, 30(4), 1-11.

Borges, T. F. C., Tamazato, A. P. S., \& Ferreira, M. S. C. (2015). Female Sex Hormone Therapy and Thromboembolic Phenomena: Literature Review. Revista, ciências em saúde, 5(2), 158-168. https://doi.org/10.21876/rcsfmit.v5i2.334

Braga, A. P. C. (2016). Efeitos do uso da contracepção de emergência: revisão de literatura. Monografia. Faculdade de Ciências da Educação e Saúde, Brasília.

Cavalcante, M. D. S, Soares, M. A., Feijó, C. \& Fonteneles, F. M. M. (2016). Perfil de utilização de anticoncepcional de emergência em serviços de atendimento farmacêutico de uma rede de farmácias comunitárias. Revista Eletrônica de Farmácia, 13(3), 131-139. https://oi.org/10.5216/ref.v13i3.37756

Dombrowski, J. G., Pontesll, J. A., \& Assis, W. A. L. M. (2013). Atuação do enfermeiro na prescrição de contraceptivos hormonais na rede de atenção primária em saúde. Revista Brasileira de Enfermagem, 66(6), 827-832. https://doi.org/10.1590/S0034-71672013000600003

Ferreira, A. P. C. et al. (2019). (Lack Of) Knowledge of women on the use of contraceptive methods. Journal of Nursing, 13(5), 1354-1360.

Foucquier, J.; Guedj, M. (2015). Analysis of drug combinations: current methodological landscape. Pharmacology Research \& perpectives, 3(3). https://doi: $10.1002 /$ prp2.149

Franco, M. S. et al. (2020). Sexual and reproductive health education of adolescent students. Journal of Nursing.

Gonçalves, L. F. F. et al. (2016). Promoção de saúde com adolescentes em ambiente escolar: Relato de experiência. SANARE Revista de Pplíticas Públicas, $15(2), 160-167$.

Kara, W. S. K, Benedicto, M., \& Mao, J. (2019). Knowledge, Attitude, and Practice of Contraception Methods Among Female Undergraduates in Dodoma, Tanzania. Cureus, 11(4). https:// doi:10.7759/cureus.4362.

Leite, A. A. G. R. et al. (2020). Conhecimentos, práticas e atitudes frente à anticoncepção de emergência: revisão sistemática. Psicologia Revista, 29(1), 201222. https://doi.org/10.23925/2594-3871.2020v29i1p201-222

[MELCON] Indústria Farmacêutica Melcon Do Brasil AS. (2015). Disponível em: < https://www.ache.com.br/produto/hormonais/>.

Mesquita, J. S. et al. (2017). Fatores de risco e proteção entre adolescentes em relação às DST/HIV/AIDS. Revista de Enfermagem da UFPE. 11(3), 12271233 .

Miranda, A. A. M. et al. (2016). Conhecimentos acerca de DST/AIDS e métodos contraceptivos dos discentes dos cursos técnicos integrados do IF Sudeste MG- Campus Juiz de Fora. Multiverso, 1(1), 25-36. 
Moura, J. R. A, Figueiredo, I. G. A, Santos, T. N. C., Sousa, E. C., Vieira, T. F., \& Lima, S. E. A. (2015). Conversas de adolescentes sobre drogas e sexualidade: um relato de experiência. Revinter, 8(2), 117-30.

Nyarko, S. H. (2015). Prevalence and correlates of contraceptive use among female adolescents in Ghana. BMC Women's Health, 15(60), 1-6. $10.1186 / \mathrm{s} 12905-015-0221-2$.

PAN American Health Organization, World Health Organization. (2018). Latin America and the Caribbean have the second highest adoescent pregnancy rates in the world.

Portela, N. L. C., Araújo, L. P. (2014). Adolescência: fontes de informações sobre métodos contraceptivos. Revista de Enfermagem da UFPI, 3(1), 93-99. DOI: https://doi.org/10.26694/reufpi.v3i1.1362

Queiroz, M. V. O., Vasconcelos, M. M., Alcântara, C. M.; Moura, M. C.; Silva, A. N. S. (2017). Características sociodemográficas e gineco-obstétricas de adolescentes assistidas em serviço de planejamento familiar. Revista de Enfermagem da UFSM, 7(4), 615-628. https://doi.org/10.5902/2179769226988

Ramos, L., Pereira, E., Lopes, K., de Araujo Filho, A., \& Lopes, N. (2018). Uso De Métodos Anticoncepcionais Por Mulheres Adolescentes De Escola Pública. Cogitare Enfermagem, 23(3). doi:http://dx.doi.org/10.5380/ce.v23i3.55230

Rodrigues, M. P. et al. (2017). Percepção sobre os efeitos psicossociais da gravidez na adolescência no cenário da estratégia e saúde da família. Revista Ciência Plural, 1(3), 81-97.

Silva, K. R. et al. (2016). Planejamento Familiar: importância das práticas educativas em saúde para jovens e adolescentes na Atenção Básica. Revista Eletrônica Gestão \& Saúde. 7(1), 327-342.

Silva, L. V. L., Beserra, A. A., \& Santos, S. C. (2017). Conhecimento de acadêmicas de enfermagem sobre o uso da contracepção de emergência. Temas em Saúde, 17(2), 61-79.

Sousa, I. C. A.; \& Álvares, A. C. M. (2018). A trombose venosa profunda como reação adversa do uso contínuo de anticoncepcionais orais. Revista Cientifica Sena Aires, 7(1), 54-65.

Spindola, T. et al. (2020). Dialogging with university students on the prevention of sexually transmitted infections; Brazilian Journal of health Review, 3(2), $2612-2621$.

Thapa, P.; Pokharel, N., \& Shrestha, M. (2018). Knowledge, Attitude and Practices of Contraception among the Married Women of Reproductive Age Group in Selected Wards of Dharan Sub-Metropolitan City. Journal of Contraceptive Studies, 3(3), 1-8.

Todd, N.; \& Black, A. (2020). Contraception for adolescents. Journal of Clinical Research in Pediatric Endocrinology, 12(1), 28-40.

Trabbold, V. L. M., Caleiro, R. C. L, Cunha, C. F., \& Guerra, A. M. C. (2016). Concepções sobre adolescentes em situação de violência sexual. Psicologia \& Sociedade, 28(1), 74-83.

Vieira, K. J. et al. (2021). Conhecimentos de adolescentes sobre métodos contraceptivos e infecções sexualmente transmissíveis. Revista baiana de enfermagem, 35 .

WORLD HEALTH ORGANIZATION. (2018). World health statistics 2018- monitoring health for de SDGs.

Zanini, M.; Selvante, J. D. S., \& Quagliato, F. F. (2017). Uso de contraceptivos e fatores associados entre adolescentes de 15 a 18 anos de idade em Unidade de Saúde da Família. Revista De Medicina- USP, 96(1), 32-34. 